7. Fernández PMM, Gatto EM, Halfon MJ. Bioquímica de los ganglios basales. In: Micheli F, editors. Enfermedad de Parkinson y Trastornos relacionados. Enfermedad de Parkinson y trastornos relacionados, $2^{\mathrm{a}}$ edición. Buenos Aires, Argentina: editorial médica Panamericana; 2006. p 47-73.

8. Kumar A, Dromerick AW. Intractable hiccups during stroke rehabilitation. Arch Phys Med Rehabil 1998;79:697-699.

Jacobo Lester, MD Neurology/Parkinson's Disease and Movement Disorders Clinic Instituto Mexicano de Neurociencias Hospital Ángeles de las Lomas Huixquilucan, Mexico State, Mexico

Gabriela Beatriz Raina, MD

Claudia Uribe-Roca, MD

Federico Micheli, MD*

Parkinson's Disease and Movement Disorders Program Hospital de Clínicas "José de San Martín"

University of Buenos Aires

Buenos Aires, Argentina

*E-mail:fmicheli@fibertel.com.ar

\section{Sparing of the Substantia Nigra in Sporadic Creutzfeldt-Jakob Disease Presenting as an Acute Corticobasal Syndrome}

Sporadic Creutzfeldt-Jakob disease (sCJD) can present with a rapidly progressive corticobasal syndrome including asymmetric parkinsonism, dystonia, apraxia, and alien limb phenomena. ${ }^{1-4}$ Loss of substantia nigra dopaminergic neurons is a requirement for the pathological diagnosis of corticobasal degeneration (CBD). ${ }^{5}$ Whether these neurons also degenerate in sCJD with acute corticobasal presentation is currently unknown. Here, we have examined the integrity of the nigrostriatal tract in a pathologically confirmed case of SCJD presenting with an acute corticobasal syndrome.

A 74-year-old right-handed man with arterial hypertension and type 2 diabetes mellitus presented to the emergency ward with a 3-week history of progressive clumsiness, stiffness and posturing of his left arm and deterioration of his vision. During the last week before admission, his gait had become slow and stooped, and he had developed speech and swallowing difficulties. According to the referring general practitioner, his mental status was still intact. There was no history of neurological diseases in his family. On examination, the patient was alert and oriented to time and place and had good insight into his medical problem. Speech was comprehensible though unclear and extremely slow. The patient was able to distinguish light from darkness, but was otherwise blind. Pupillary reflexes to light were preserved. Extensive testing of cognitive functions was difficult due to the slowness of speech and the visual deficit, but there was no obvious memory or language impairment. The patient had a tendency to deviate his head and gaze to the right. Facial expression was markedly reduced. There

Published online 29 May 2007 in Wiley InterScience (www. interscience.wiley.com). DOI: 10.1002/mds.21570 were blepharospasms. There was a striking dystonic flexion posture and action myoclonus of the left arm and occasional levitation of the right arm. Ideomotor apraxia was marked in the left hand and mild in the right hand. There was rigidity in the four limbs, especially on the left side. Strength and sensation were normal. Tendon reflexes were increased bilaterally, with a Babinski sign on the left. There was no tremor or ataxia. Gait was profoundly hypokinetic, with short steps, stooped posture, prominent freezing, and postural instability. Over the next few weeks, the patient developed anarthria, generalized dystonia and myoclonus, and incontinence. The patient died of pneumonia 4 weeks after admission.

T2- and diffusion-weighted MRI on the day of admission revealed normal appearance of the basal ganglia and bilateral hyperintensity of the parietal and occipital cortex, especially on the right side (Fig. 1A). EEGs showed generalized triphasic complexes. Routine blood, urine, and CSF investigations for infectious, inflammatory, toxic, or metabolic causes were negative. CSF 14-3-3 protein was positive. Seven days after admission, ${ }^{99 \mathrm{~m}} \mathrm{Tc}-\mathrm{ECD}$ SPECT scan showed pronounced hypoperfusion over the occipital, parietal, and lateral temporal cortex of the right hemisphere and, to a lesser extent, the left parietal cortex (Fig. 1B). Semiquantitative ${ }^{123}$ I-FP-CIT SPECT imaging of the dopamine transporter showed normal striatal ${ }^{123}$ I-FP-CIT binding compared with published results from 27 patients with essential tremor ${ }^{6}$ (Fig. 1C).

Upon pathological examination, the brain weighed $1,300 \mathrm{~g}$. Macroscopic inspection of the brain showed generalized atrophy of the cerebral cortex and normal pigmentation of the substantia nigra. Microscopy revealed widespread spongiform changes and gliosis in the cerebral (especially occipital) cortex (Fig. 1D), caudate nucleus, putamen (Fig. 1E), and the molecular layer of the cerebellar cortex, as well as synaptic-type abnormal prion protein $\left(\mathrm{PrP}^{\mathrm{sc}}\right)$ deposits in these areas. ${ }^{7} \mathrm{Re}-$ markably, none of these pathological abnormalities were found in the substantia nigra (Fig. 1F).

Although the asymmetric dystonia, myoclonus, parkinsonism, apraxia, and levitation of this patient were reminiscent of $\mathrm{CBD}$, the rapid disease progression and the cortical visual deficit strongly suggested the diagnosis of CJD, which was supported by MRI, EEG, and CSF 14-3-3 analysis and confirmed by neuropathological examination. Histological abnormalities of the substantia nigra are considered uncommon in the more typical form of CJD presenting as a rapidly progressive dementing illness. ${ }^{8,9}$ Whether relative sparing of the nigrostriatal tract also occurs in CJD with acute corticobasal presentation has not been addressed before.

Unexpectedly, antemortem ${ }^{123}$ FP-CIT SPECT imaging and postmortem histological examination revealed remarkable preservation of nigral dopaminergic neurons in our patient, despite the clinical similarities with CBD, a disease in which nigrostriatal cell loss is an invariable pathological feature. ${ }^{5}$ The striking neuropathological changes in caudate and putamen suggest the possibility that the patient's parkinsonism may have been caused by dysfunction of the nigrostriatal system at the postsynaptic level. Alternatively, the parkinsonian features may have been due to degeneration of other neuronal circuits. Interestingly, Boeve and colleagues reported a case of CJD with corticobasal presentation and a much more chronic disease course (4 years). ${ }^{10}$ Neuropathological examination of their case showed sparing of the substantia nigra, as in our patient. However, in contrast to our case, the striatum was also pre- 

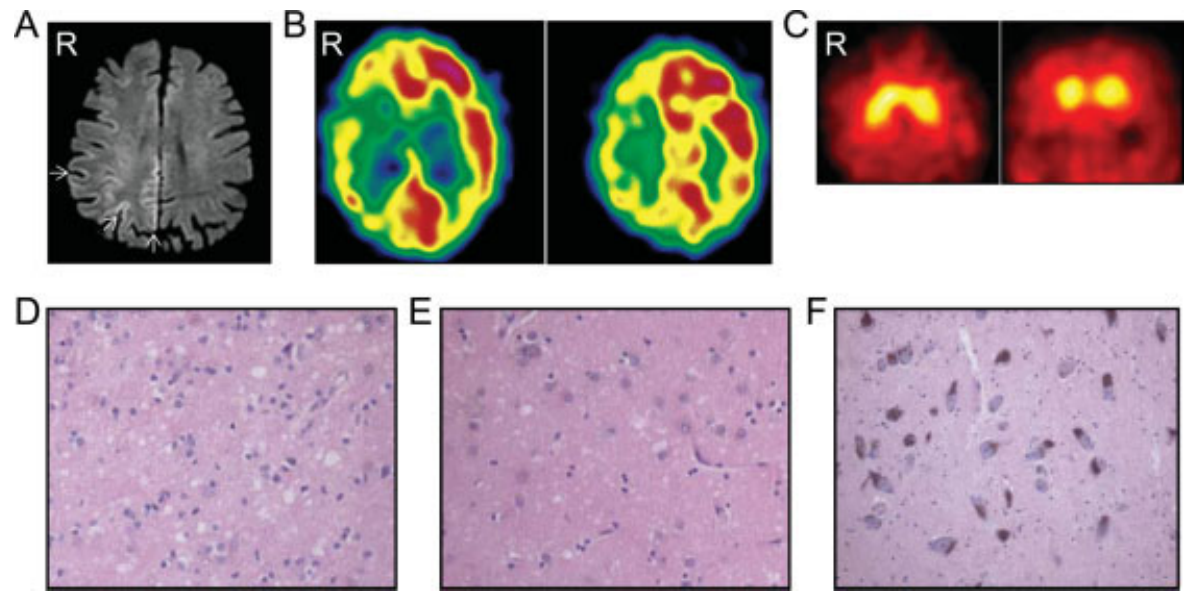

FIG. 1. A: Diffusion-weighted MRI shows hyperintensity of the right parietal cortex (arrows). B: Representative axial ${ }^{99 \mathrm{~m}} \mathrm{Tc}-\mathrm{ECD}$ SPECT images show pronounced hypoperfusion over the right cerebral cortex. C: Axial (left panel) and coronal (right panel) SPECT images show normal striatal ${ }^{123}$ I-FP-CIT binding. D-F: Hematoxylin-eosin stain of sections of occipital cortex (D), putamen (E), and substantia nigra (F). Original magnifications are $\times 50(\mathrm{D}, \mathrm{E})$ and $\times 400$ (F). served, suggesting that parkinsonism in the "corticobasal" form of CJD may indeed originate in structures outside the basal ganglia, such as cerebral cortex or subcortical white matter.

Acknowledgments: We thank Dr. Herbert Budka (Medical University Vienna) for PrP immunohistochemistry. W.V. and K.V.L. are Senior Clinical Investigators of the Fund for Scientific Research - Flanders.

Wim Vandenberghe*
Department of Neurology, University Hospital Gasthuisberg,
Leuven, Belgium
*E-mail: wim.vandenberghe@uz.kuleuven.ac.be

Raf Sciot

Department of Pathology, University Hospital Gasthuisberg, Leuven, Belgium

Philippe Demaerel Department of Radiology, University Hospital Gasthuisberg, Leuven, Belgium

Koen Van Laere Department of Nuclear Medicine, University Hospital Gasthuisberg, Leuven, Belgium

\section{References}

1. Anschel DJ, Simon DK, Llinas R, Joseph JT. Spongiform encephalopathy mimicking corticobasal degeneration. Mov Disord 2002; 17:606-607.

2. Kleiner-Fisman G, Bergeron C, Lang AE. Presentation of Creutzfeldt-Jakob disease as acute corticobasal degeneration syndrome. Mov Disord 2004;19:948-949.

3. Moreaud O, Monavon A, Brutti-Mairesse MP, Grand S, Lebas JF Creutzfeldt-Jakob disease mimicking corticobasal degeneration. Clinical and MRI data of a case. J Neurol 2005;252:1283-1284.

4. Fogel B, Wu M, Kremen S, Murthy K, Jackson G, Vanek Z. Creutzfeldt-Jakob disease presenting with alien limb sign. Mov Disord 2006;21:1040-1050.

5. Dickson DW, Bergeron C, Chin SS, et al. Office of Rare Diseases neuropathological criteria for corticobasal degeneration. J Neuropathol Exp Neurol 2002;61:935-946.

6. Van Laere K, Casteels C, De Ceuninck L, et al. Dual-tracer dopamine transporter and perfusion SPECT in differential diagno- sis of parkinsonism using template-based discriminant analysis. J Nucl Med 2006;47:384-392.

7. Kovacs GG, Head MW, Hegyi I, et al. Immunohistochemistry for the prion protein: comparison of different monoclonal antibodies in human prion disease subtypes. Brain Pathol 2002;12:1-11.

8. Budka H, Aguzzi A, Brown P, et al. Neuropathological diagnostic criteria for Creutzfeldt-Jakob disease (CJD) and other human spongiform encephalopathies (prion diseases). Brain Pathol 1995;5: 459-466.

9. Zeidler M, Gibbs CJ, Jr, Meslin F. WHO manual for strengthening diagnosis and surveillance of Creutzfeldt-Jakob disease. Geneva: World Health Organization; 1998. p 47-51.

10. Boeve BF, Maraganore DM, Parisi JE, et al. Pathologic heterogeneity in clinically diagnosed corticobasal degeneration. Neurology 1999;53:795-800.

\section{A New Phenotype of Chorea-Acanthocytosis with Dilated Cardiomyopathy and Myopathy}

Chorea-acanthocytosis (ChAc) and McLeod syndrome $(\mathrm{McS})$ are uncommon hereditary diseases, associated with acanthocytosis. Cardiomyopathy and myopathy have been described as specific features only of McS and not seen in ChAc. ${ }^{1}$ We report a genetically proved $\mathrm{ChAc}$ case with dilated cardiomyopathy and myopathy, clinically undistinguishable from McS.

\section{CASE REPORT}

A 40-year-old male was referred for progressive gait disturbance followed by generalized involuntary movements. Neurological examination showed generalized chorea, tic, and truncal dystonia. Mild muscular atrophy and weakness were observed in lower limbs. Serum creatine kinase was elevated to $5.514 \mathrm{IU} / \mathrm{l}$ (normal values, 0-160) and approximately 5.6\% acanthocytes were detected.

Published online 21 May 2007 in Wiley InterScience (www. interscience.wiley.com). DOI: 10.1002/mds.21556 